\section{BIOLOGICAL MECHANISMS IN THE DIFFERENT ETIOLOGIES OF MERKEL CELL CARCINOMA PATIENTS: POLYOMAVIRUS OR UV EXPOSURE}

${ }^{1}$ Domenico Mallardo*, 'Giosuè Scognamiglio, ${ }^{2}$ Khrystyna North, ${ }^{1}$ Mariaelena Capone, ${ }^{2}$ Michael Bailey, ${ }^{3}$ Luigi Scarpato, ${ }^{2}$ Sarah Church, ${ }^{1}$ Gabriele Madonna, ${ }^{2}$ Jason Reeves, ${ }^{1}$ Marcello Curvietto, ${ }^{1}$ Marilena Tuffanelli, ' ${ }^{1}$ grazia d'angelo, ${ }^{1}$ Ester Simeone, 'Lucia Festino, 'Vito Vanella, ${ }^{1}$ Claudia Trojaniello, 'Maria Grazia Vitale, 'Salvatore Tafuto, ${ }^{1}$ Corrado Caraco, ${ }^{1}$ Anna Maria Anniciello, ${ }^{1}$ Nicola Normanno, ${ }^{1}$ Maurizio Di Bonito, ${ }^{2}$ Sarah Warren, ${ }^{1}$ Paolo Ascierto. ${ }^{1}$ Istituto Nazionale Tumori IRCCS Pascale, Naples, Italy; ${ }^{2}$ NanoString Technologies, Seattle, WA, USA; ${ }^{3}$ stituto Nazionale Tumori IRCCS Pascalel, Naples, Italy

Background Merkel cell carcinoma (MCC) is a rare and aggressive skin cancer with neuroendocrine features, and it is associated with elevated mortality. The pathogenesis is associated with presence of clonally integrated Merkel cell polyomavirus (MCPyV) or ultraviolet light (UV) exposure. ${ }^{1}$ The MCPyV causes up to $80 \%$ of MCC tumors in North America and Europe. ${ }^{2-4}$ Recently immunotherapy is having good results, ${ }^{5}$ the phase 2 trial JAVELIN Merkel 200 indicated that treatment with Avelumab (PDL1 inhibitor) in patients with metastatic MCC pre-treated have a meaningful long-term survival outcomes respect chemotherapy. Moreover, ORRs were highest in patients with high TMB that were also MCPyV-, $\mathrm{PD}-\mathrm{L} 1+$ or had a greater CD8 $+\mathrm{T}$ cell density at the invasive margin. ${ }^{6}$ In this study, we investigated the biological signatures in patients with MCPyV or not.

Methods From April 2011 to June 2018, we collected retrospectively 50 FFPE (Formalin-Fixed Paraffin-Embed) from 37 patients with metastatic MCC and 13 tissues from a secondary metastatic site. All patients have appropriately signed informed consent. We performed an immunohistochemistry assays (IHC) for MCPyV and PDL1. In addition, through the NanoString GeoMx DSP (Digital Spatial Profiling), we analysed 11 patients (6 MCPyV+; $5 \mathrm{MCPyV}$-) with cutaneous metastasis using a 44-plex antibody cocktail. For each slide we selected three different areas: Intratumoral, extratumoral and tumour border, in each area we selected CD4+ and CD8+ cells in 4 different ROIs (Region of Interest). Statistical analysis was performed via Bonferroni correction, $\mathrm{P}<0.05$ was considered statistically significant for median stratification.

Results The DSP analysis showed that the tumour border cells have an overexpression of IDO respect intratumoral area (adj. $\mathrm{p}<0.01$ ). Instead, extratumoral area of $\mathrm{MCPyV}$ - patients have a higher expression of $\mathrm{B} 7-\mathrm{H} 3$ respect $\mathrm{MCPyV}+$ as well as FOXP3 is higher in the tumour border of $\mathrm{MCPyV}+$ patients and EpCAM in the intratumoral area $(\mathrm{p}<0.05)$. PDL1 is overexpressed in $\mathrm{MCPyV}+\mathrm{CD} 4+$ cells respect CD8 $+(\mathrm{p}<0.05)$. The IHC assay shown that viral status does not change in multiple metastases and PDL1 is elevated in the tumour bor$\operatorname{der}(\mathrm{p}<0.05)$.

Conclusions In this retrospective study, our preliminary data shown that tumour edge have an important role in the modulations of immune infiltrate and patients with Merkel cell polyomavirus could have a different pathway of immunosuppression compared to patients with non-virus related etiology. Further investigations are needed to get additional information.

Acknowledgements The study was supported by the Institutional Project "Ricerca Corrente" of Istituto Nazionale Tumori IRCCS Fondazione "G. Pascale" of Napoli, Italy.

\section{REFERENCES}

1. Kaae J, Hansen AV, Biggar RJ, et al. Merkel cell carcinoma: incidence, mortality, and risk of other cancers. J Nat/ Cancer Inst 2010 June 2;102(11):793-801.

2. Feng $\mathrm{H}$, Shuda $\mathrm{M}$, Chang $\mathrm{Y}$, et al. Clonal integration of a polyomavirus in human Merkel cell carcinoma. Science 2008 February 22;319(5866):1096-100.

3. Garneski KM, Warcola AH, Feng Q, et al. Merkel cell polyomavirus is more frequently present in North American than Australian Merkel cell carcinoma tumors. Invest Dermatol 2009 January:129(1):246-8.

4. Goh G, Walradt T, Markarov V, et al. Mutational landscape of MCPyV-positive and MCPyV-negative Merkel cell carcinomas with implications for immunotherapy. Oncotarget 2016 January 19;7(3):3403-15.

5. Bichakjian CK, Olencki T, Aasi SZ, et al. Merkel cell carcinoma, version 1.2018 , NCCN Clinical Practice Guidelines in Oncology. J Natl Compr Canc Netw 2018 June; $16(6): 742-774$

6. D'Angelo SP, Bhatia S, Brohl AS, et al. Avelumab in patients with previously treated metastatic Merkel cell carcinoma: long-term data and biomarker analyses from the single-arm phase 2 JAVELIN Merkel 200 trial. J Immunother Cancer 2020 May;8(1):e000674.

Ethics Approval The study was approved by internal ethics board of the Istituto Nazionale Tumori IRCCS Fondazione "G. Pascale" of Napoli Italy, approval number of registry 33/ 17 OSS.

Consent Written informed consent was obtained from the patient for publication of this abstract and any accompanying images. A copy of the written consent is available for review by the Editor of this journal.

http://dx.doi.org/10.1136/jitc-2021-SITC2021.934 